\title{
Manejo del dolor neuropático: la gabapentina como opción
}

\author{
R. de Dios del Valle, M. González Torrejón*, \\ A. SACRISTÁn RODEA* \\ Residente en $3^{\star}$ año de Mediana de Familia y Comunitaria. C.S. Dr. Cirajas. Área 4. \\ Madrid. * Médico de la Unidad de Cuidados Paliativos del Área 4. Madrid
}

\section{RESUMEN}

El dolor es un síntoma muy frecuente en las consultas de Atención Primaria. Podemos dife renciar dos tipos de dolor, dolor nociceptivo y do lor neuropático; éste último fue considerado du rante mucho tiempo como un dolor intratable y aún en la actualidad se controla con mayor difi cultad que el dolor nociceptivo. Existen diferentes medicamentos, antidepresivos y anticonvulsivan tes principalmente, utilizados en el tratamiento de este tipo de dolor, aunque como veremos existen pocas evidencias en la actualidad que nos orien ten sobre cómo manejar a estos pacientes.

Presentamos un caso clínico de un paciente diagnosticado de cordoma con objeto de ejempli ficar el manejo terapéutico del dolor neuropático, para el que no existen protocolos clínicos de ac tuación consensuados. Veremos como se usaron diferentes medicamentos, de los que habitualmen te se utilizan en el manejo de este dolor, sin lograr controlar el dolor. En nuestro caso la gabapenti na, fármaco poco usado como analgésico, fue de utilidad para el control del dolor neuropático.

Palabras clave: Dolor. Cuidados Paliativos. Anticonvulsivantes.
Neuropathy pain treatment: gabapentin is an option

\begin{abstract}
The painful is a common sympton in Primary Health Care consultation. It is possible to find two sorts of painful; the nociceptive and the neuro pathy one. The neuropathy pain has often been considered as a pain without treatment, and ac tually it is more difficult to control than de noci ceptive pain. There are different drugs, specially antidepresive and antiepileptic drugs, used in the neuropathy pain treatment, but there are few evi dences and no clinical protocols about which is the best management of pain in these patients.

It is reported a case of a patient with cordoma as example of therapeutic management of neuro pathy pain, the use of different drugs without suc ces. In this case, the gabapentin, drug not very used as analgesic, was useful in the control of the neuropathy pain.
\end{abstract}

Key words: Pain. Palliative Care. Anticonvul sants.

es diferente. El dolor nociceptivo depende de la estimulación de receptores nociceptivos a nivel cutáneo, visceral y óseo. Ante determinados estímulos se produce la activación de estos receptores y la transmisión del impulso eléctrico a través de las vías aferentes (fibras A-delta y C), por las raíces dor- 
sales de la médula hasta los cuernos dorsales llegando al haz espino-talámico anterolateral del tálamo y córtex donde es interpretado como un estímulo doloroso. Este dolor se puede subclasificar en dolor somático y dolor visceral. El primero se produce por el estímulo de receptores a nivel de piel, periostio, peritoneo y pleura. El paciente describe este dolor como continuo, punzante y bien localizado. En el dolor visceral se produce el estímulo doloroso en los órganos inervados por el sistema simpático, siendo descrito por el paciente como un dolor sordo y profundo, mal localizado y a menudo descrito como dolor referido. El dolor neuropático se produce por lesión (compresión o infiltración) de nervios periféricos o centrales. No existe un estímulo nociceptivo claro, estando el dolor pobremente localizado y siendo de carácter disestésico. El paciente suele presentar un dolor continuo y quemante, presentando reagudizaciones paroxísticas de dolor generalmente asociado a disfunción sensitivo-motora de la zona afectada. El dolor neuropático fue considerado durante mucho tiempo como un dolor intratable, ya que no respondía a los tratamientos analgésicos habituales. El ejemplo típico de este tipo de dolor es la neuralgia del trigémino ${ }^{1}$.

Presentamos un caso de un paciente diagnosticado de cordoma que se queja de dolor continuo y quemante, presentando reagudizaciones paroxísticas y sensación disestésica de la zona afectada, como ejemplo de manejo de dolor neuropático. El uso de fármacos anticonvulsivantes para el control del dolor neuropático es aún controvertido existiendo pocas evidencias en la literatura médica sobre el tema, así es difícil encontrar citas bibliográficas relevantes sobre el tema.

El cordoma es una neoplasia generada a partir de restos de la notocorda en la línea media del eje neural y que afecta el hueso adyacente. Histológicamente la célula fisalífera es patognomónica. Este tumor es muy maligno debido al alto índice de recidivas y complicaciones locales. La muerte del paciente se debe mayoritariamente a las manifestaciones locales. La supervivencia media es de 5,7 años. La manifestación clínica más frecuente es un dolor sordo y ocasionalmente estreñimiento. El tratamiento consiste en intervención quirúrgica, siendo la primera la que gozará de mayores probabilidades de éxito ${ }^{2}$.

\section{CASO CLÍNICO}

\section{Historia oncológica}

Varón de 57 años. Sin antecedentes personales de interés. En julio de 1998 comienza a presentar un dolor lumbar intenso que se irradia a miembro inferior derecho. Se le comienza a tratar con antiinflamatorios no esteroideos (AINEs) y relajantes musculares sin responder al tratamiento. En septiembre del mismo año se le realiza una resonancia magnética nuclear donde se evidencia una lesión en la $12^{\mathrm{a}}$ vértebra torácica; con posterioridad se somete a una punción aspiración dirigida con TAC de la lesión dando una celularidad compatible con cordoma.

En enero de 1999 se somete a una intervención quirúrgica de la lesión, realizándose extirpación quirúrgica de la misma, colocación de un injerto de diáfisis femoral de donante e instrumentación anterior y posterior. La anatomía patológica de la pieza quirúrgica confirma el diagnóstico de cordoma. En julio de 1999 recibe radioterapia postoperatoria. En diciembre de 1999 en un TAC de control se evidencia una masa paravertebral con desplazamiento del riñon derecho, afectación del espacio paravertebral y paraespinal posterior compatible con recidiva. En enero de 2000 recibe nuevamente radioterapia con intención paliativa (dosis total $50 \mathrm{~Gy}$ ). Debido al deterioro clínico del enfermo y a la escasa respuesta al tratamiento se suspende la radioterapia habiéndose aplicado una dosis acumulada de $40 \mathrm{~Gy}$.

\section{Historia actual}

Varón de 57 años que es derivado en enero de 2000 a la Unidad de Cuidados Paliativos del Área 4 de Madrid. El paciente presenta en ese momento intenso dolor quemante y continuo en región lumbar que irradia a miembro inferior derecho, disminución de la fuerza en ese miembro. Se acompaña de estreñimiento y retención urinaria. A la exploración presenta dolor severo a la palpación a nivel de columna lumbar (lugar de la intervención), dolor a la movilización de la región en todos los planos. Movilidad limitada de las extremidades inferiores por el dolor. Resto de la exploración física dentro de la normalidad. Karnofsky de $40 \%$. Desde hace unos meses el paciente presenta un estado de ánimo ansioso-depresivo en relación con su estado de salud. En ese momento el paciente estaba siendo tratado con sulfato de morfina $120 \mathrm{mg}$ por la mañana, $60 \mathrm{mg}$ al mediodía y $120 \mathrm{mg}$ por la noche, ranitidina $150 \mathrm{mg}$ cada 12 horas, lorazepam 1 mg cada 8 horas, amitriptilina $25 \mathrm{mg}$ cada 24 horas, dexametasona $2 \mathrm{mg}$ por la mañana y $2 \mathrm{mg}$ al mediodía y solución oral de morfina $40 \mathrm{mg}$ cada 4 horas.

\section{Evolución}

El paciente continúa presentando dolor de las mismas características, con incremento de su inten- 
sidad hasta hacerse de reposo, siendo insoportable a la movilización y persistente en reposo sin ceder con el tratamiento analgésico a pesar del incremento en la dosis de amitriptilina hasta $75 \mathrm{mg}$ al día. En marzo de ese mismo año se suspende la amitriptilina debido a la presencia de efectos anticolinérgicos intolerables y no haberse logrado una respuesta analgésica adecuada, llegando el paciente a estar encamado durante todo el día. En este momento se readapta la pauta de morfina y se introduce carbamazepina $200 \mathrm{mg}$ cada 12 horas. Debemos suspender el tratamiento con carbamazepina por la aparición de cuadros vertiginosos como efecto secundario. En abril de 2000 se introduce paroxetina $20 \mathrm{mg}$ al día con objeto de tratar la sintomatología depresiva, y gabapentina $300 \mathrm{mg}$ cada 8 horas llegando a una dosis máxima de $1.200 \mathrm{mg} /$ día como tratamiento para el dolor neuropático. Díez días después de alcanzar esta dosis de gabapentina se produce una buena respuesta sintomática con adecuado control del dolor llegando a ser nulo tanto en reposo como a la movilidad. Durante estos meses se ha ido reduciendo la dosis de dexametasona, no pudiéndose retirar por reaparición del dolor, en el momento actual está con una dosis de dexametasona de $2 \mathrm{mg}$. La situación actual del paciente es ausencia de dolor en reposo, realiza paseos de $2 \mathrm{~km}$ al día y presenta clara mejoría del estado anímico. Karnofsky en este momento de $60 \%$, lo cual implica que pasa de una situación de estar imposibilitado y requerir cuidado especial y asistencia a una situación en que requiere asistencia ocasional, haciéndose cargo de la mayoría de sus necesidades.

\section{DISCUSIÓN}

El dolor neuropático fue considerado durante mucho tiempo como intratable ya que era resistente a los fármacos analgésicos habituales (AINEs y opiáceos). En la actualidad se prescriben esteroides, antidepresivos y anticonvulsivantes para el control de este tipo de dolor. No existen protocolos que nos indiquen qué fármacos usar de primera elección en el tratamiento de este dolor, como orientación podemos exponer la siguiente tabla (Tabla I).

En este caso el dolor es continuo y de carácter disestésico, lo cual nos hizo comenzar con corticoterapia con objeto de reducir el componente compresivo y con antidepresivos tricíclicos, no logrando una adecuada respuesta terapéutica. Como medicamento de segunda elección se encuentran los anticonvulsivantes. En nuestro paciente utilizamos carbamazepina, teniendo que ser suspendida debido a la aparición de efectos secundarios. Como alternativa empleamos la gabapentina. Con la combinación de esteroides y gabapentina se ha conse-

\begin{tabular}{|c|c|c|}
\hline \multicolumn{3}{|c|}{ TRATAMIENTO DEL DOLOR NEUROPÁTICO } \\
\hline $\begin{array}{l}\text { Dolor } \\
\text { neuropático }\end{array}$ & Primera línea & Segunda línea \\
\hline Continuo & $\begin{array}{l}\text { A.D. tricíclicos: PO } \\
\text { - Amitriptilina: } \\
\text { 25-300 mg/ } 24 \text { horas } \\
\text { - Imipramina: } \\
\text { igual dosis }\end{array}$ & Anticonvulsivantes \\
\hline Lacinante & $\begin{array}{l}\text { Anticonvulsivantes: PO } \\
\text { - Carbamazepina: } \\
\text { 100-200 mg } \\
\text { cada } 12 \text { horas }\end{array}$ & A.D. tricíclicos \\
\hline Compresión & $\begin{array}{l}\text { Esteroides: } 10 \text { días } \\
\text { - Dexametasona: } \\
60 \text { mg/ } 24 \text { horas IV } \\
\text { - Metilprednisolona: } \\
125 \text { mg/ } 24 \text { horas IM }\end{array}$ & Analgésicos opiáceos \\
\hline $\begin{array}{l}\text { A.D.: antide } \\
\text { IM: intramus }\end{array}$ & $\begin{array}{l}\text { ivos; PO: posología } \\
\text { r; h: horas; mg: miligran }\end{array}$ & $\begin{array}{l}\text { al; IV: intravenosa; } \\
\text { s. }\end{array}$ \\
\hline
\end{tabular}

guido un buen control del dolor, mejorando el estado funcional del paciente y su estado de ánimo. El uso de fármacos anticonvulsivantes en el tratamiento del dolor se remonta a la década de los 60 . La mayor parte de los ensayos clínicos sobre el tratamiento del dolor neuropático se centra en casos de neuralgia del trigémino, neuralgia post-herpética y neuropatía diabética ${ }^{3}$. En una revisión realizada por la fundación Cochrane ${ }^{4}$ sobre el uso de anticonvulsivantes en el tratamiento del dolor, se concluye que son necesarios más estudios para aportar evidencias suficientes sobre la conveniencia del uso de fármacos anticonvulsivantes en el tratamiento del dolor, confirman que el fármaco con el que se posee mayor experiencia es la carbamazepina, fundamentalmente en el tratamiento de la neuralgia del trigémino donde ha demostrado mayor eficacia que el placebo ${ }^{5,6}$. Ningún estudio de esta revisión utiliza la gabapentina. Un ensayo clínico randomizado ${ }^{7}$ en el que se determina la eficacia del tratamiento con gabapentina en pacientes con neuralgia post-herpética nos muestra que el grupo tratado con gabapentina presenta una reducción estadísticamente significativa del dolor mayor que el grupo control, incluso en pacientes que han sido tratado previamente con carbamazepina sin éxito. La dosis de inicio de gabapentina en dicho estudio es de $300 \mathrm{mg}$ aumentando hasta una dosis máxima de $3.600 \mathrm{mg}$. No existen estudios comparativos entre diferentes fármacos anticonvulsivantes, ni entre éstos y fármacos antidepresivos. Debemos decir que no existen ensayos clínicos que valoren la eficacia de estos fármacos en el tratamiento del dolor neuropático de origen oncológico. 
Este caso apunta a la gabapentina como un fármaco de utilidad en el tratamiento del dolor neuropático, aunque seguimos considerando que los antidepresivos y la carbamazepina son los fármacos de primera elección según las características del dolor.

Debemos exponer que en nuestro caso pensamos que el dolor presenta un componente mixto, de tipo nociceptivo debido a la gran masa presente que infiltra diferentes estructuras y por otro lado neuropático. Este hecho nos hace tratar al paciente con fármacos dirigidos a controlar el dolor nociceptivo como la morfina y medicamentos que nos ayuden a controlar el dolor neuropático como la gabapentina. Debemos decir que probablemente los corticoides han jugado un papel esencial en la evolución del paciente reduciendo el efecto compresivo. Llegados a este punto queremos introducir una reflexión acerca de la pauta de opiáceos que este paciente tenía en un principio. Debemos recordar que la morfina por vía oral existe en forma de liberación rápida y en comprimidos de liberación sostenida, la duración de la analgesia es de 4 horas para los primeros y de 12 horas para los segundos. En un primer momento se suele titular la dosis diaria de morfina con compuestos de liberación rápida, con dosis repetidas para posteriormente pasar a compuestos de liberación retardada. Para los pacientes tratados que presentan reagudizaciones ocasionales de dolor (picos de dolor) podemos utilizar una dosis extra de morfina de liberación rápida. En este caso se realizó una readecuación de la pauta de morfina con $100 \mathrm{mg}$ cada 12 horas de sulfato de morfina de liberación controlada, sin requerir dosis suplementarias de morfina de liberación rápida.

\section{CORRESPONDENCIA:}

R. de Dios del Valle

C/ San Modesto 42, $4^{\circ} \mathrm{A}$

28034 Madrid

Tfno.: 913855545

\section{Bibliografía}

1. Lobera L, González Torrejón M. Estrategia farmacológica para el tratamiento del dolor crónico en el cáncer. Información Terapéutica del Sistema Nacional de Salud 1995; 19 (6): 129-39.

2. De Vita V, Hellman S, Rosenberg S. Cáncer. Principios y Práctica de Oncología (2a ed.). Barcelona: Salvat, 1988; 1244-5.

3. McQuay H, Carroll D, Jadad AR, Wiffen P, Moore A. Anti convulsant drugs for management of pain: a systematic review. BMJ 1995; 311 (7012): 1047-52.

4. Wiffen P, McQuay H, Carroll D, Jadad A, Moore A. Anti- convulsant drugs for acute and chronic pain. Cochrane Library 2000; CD001133.

5. Killian JM, Fromm GH. Carbamazepine in the treatment of neuralgia. Use of side effects. Arch Neurol 1968; 19: 12936.

6. Nicol CF. A four year double blind study of tegretol in facial pain. Headache 1969; 9: 54-7.

7. Rowbotham M, Harden N, Stacey B, Bernstein P, MagnusMiller L, for the Gabapentin Postherpetic Neuralgia Study Group. Gabapentin for the treatment of post-herpetic neuralgia: a randomized controlled trial. JAMA 1998; 280: 1837-42. 